\title{
Review
}

\section{The diagnosis and management of pre-invasive breast disease Flat epithelial atypia - classification, pathologic features and clinical significance}

Stuart J Schnitt

\author{
Department of Pathology, Beth Israel Deaconess Medical Center and Harvard Medical School, Boston, Massachusetts, USA \\ Correspondence: Stuart J Schnitt (e-mail: sschnitt@bidmc.harvard.edu)
}

Published: 29 July 2003

Breast Cancer Res 2003, 5:263-268 (DOI 10.1186/bcr625)

(C) 2003 BioMed Central Ltd (Print ISSN 1465-5411; Online ISSN 1465-542X)

\begin{abstract}
Flat epithelial atypia is a descriptive term that encompasses lesions of the breast terminal duct lobular units in which variably dilated acini are lined by one to several layers of epithelial cells, which are usually columnar in shape and which display low-grade cytologic atypia. Observational studies have suggested that at least some of these lesions may represent either a precursor of ductal carcinoma in situ (DCIS) or the earliest morphological manifestation of DCIS. In contrast, the limited available clinical follow-up data suggest that the risk of both local recurrence and progression of these lesions to invasive cancer is extremely low, supporting the notion that categorizing such lesions as 'clinging carcinoma' and managing them as if they were fully developed DCIS will result in overtreatment of many patients. Additional studies are needed to better understand the biological nature and clinical significance of these lesions.
\end{abstract}

Keywords: atypia, breast, columnar cell, ductal carcinoma in situ, flat

\section{Introduction}

Mammographic screening has resulted in the increased detection of invasive breast cancers as well as the detection of certain lesions regarded to be precursors of invasive breast cancer (such as ductal carcinoma in situ [DCIS]) and risk factors for the subsequent development of breast cancer (such as atypical ductal hyperplasia $[A D H])$. In addition, several breast lesions that were only uncommonly seen in the premammographic era are currently being encountered with increasing frequency in breast biopsies performed because of a mammographic abnormality, particularly microcalcifications. Included within this group are lesions that have recently been designated 'flat epithelial atypia' by the World Health Organization Working Group on the Pathology and Genetics of Tumours of the Breast [1].

\section{Classification and pathologic features}

'Flat epithelial atypia' is better considered a descriptive term that encompasses several different lesions, rather than a specific pathologic diagnosis per se. Despite the diversity of published descriptions and the wide assortment of names applied to breast lesions that would currently be included in the category of flat epithelial atypia [2-11] (Table 1), for practical purposes these lesions can be grouped into one of two diagnostic categories: columnar cell change with atypia or columnar cell hyperplasia with atypia [12]. Although 'flat epithelial atypia' will be used for purposes of discussion in the present review, we use the more specific diagnostic terms 'columnar cell change with atypia' and 'columnar cell hyperplasia with atypia' in clinical practice and in surgical pathology reporting.

\section{Columnar cell change with atypia}

Columnar cell change with atypia is characterized by terminal duct lobular units that display variably dilated acini lined by one or two layers of epithelial cells that are columnar in shape, although the height of these columnar cells varies. Apical cytoplasmic blebs or snouts are often present at the luminal surface of the epithelial cells and, in 
Table 1

\begin{tabular}{lr}
$\begin{array}{l}\text { Other names used to describe lesions within the category of } \\
\text { flat epithelial atypia }\end{array}$ & Reference \\
\hline Description & {$[2]$} \\
\hline Atypical cystic duct & {$[3]$} \\
Atypical cystic lobules & {$[4]$} \\
Atypical lobules type A (some examples) & {$[5,6]$} \\
Clinging carcinoma (monomorphic type) & {$[7]$} \\
Columnar alteration with prominent apical snouts and & {$[12]$} \\
secretions with atypia & {$[12]$} \\
Columnar cell change with atypia & {$[8]$} \\
Columnar cell hyperplasia with atypia & \\
Ductal intraepithelial neoplasia of the flat monomorphic type & {$[9]$} \\
Hypersecretory hyperplasia with atypia (some examples) & {$[10]$} \\
Pretubular hyperplasia & {$[11]$} \\
Small ectatic ducts lined by atypical ductal cells with & \\
apocrine snouts &
\end{tabular}

aPreferred diagnostic terms.

some cases, may be prominent or exaggerated, which may impart an irregular contour to the luminal aspect of the acini.

Flocculent secretions are often present in the acinar lumina. In addition, the acini often contain luminal calcifications that may be irregular or may have the appearance of psammoma bodies (Fig. 1a). The cells lining the involved acini show cytologic atypia, which is usually of low grade. This atypia is characterized by the presence of columnar epithelial cells with round to ovoid nuclei that are not regularly oriented perpendicular to the basement membrane, with a slight increase in the nuclear/cytoplasmic ratio. The nuclear chromatin may be evenly dispersed or slightly mar- ginated, and the nucleoli are variably prominent. Mitotic figures may be seen but are uncommon (Fig. 1b). In other cases, the columnar cells may resemble the cells comprising the tubules of tubular carcinoma. The cytologic atypia in these cases may be quite subtle.

In some cases of columnar cell change with atypia, the lesion may be overlooked entirely on low-power microscopic examination due to the lack of significant cellular proliferation and the subtle nature of the cytologic atypia [8]. In fact, terminal duct lobular units exhibiting this alteration are often misinterpreted on low-power microscopic examination as either normal or as showing only microcysts. It is only after examination of such foci under high magnification that the subtle cytologic atypia becomes evident.

\section{Columnar cell hyperplasia with atypia}

The term columnar cell hyperplasia with atypia is used to describe lesions composed of terminal duct lobular units with variably dilated acini lined by columnar cells that have cytologic features similar to those seen in columnar cell change with atypia but that also show cellular stratification of more than two cell layers. The proliferating epithelial cells may form small mounds, tufts or short micropapillations. However, complex architectural patterns, such as well-developed micropapillations, rigid cellular bridges, bars and arcades, or sieve-like fenestrations, with evidence of cellular polarization within the micropapillations and bars or around the fenestrations, are absent (Fig. 2). Thus, while these lesions are included under the heading 'flat epithelial atypia', it should be apparent that 'flat' is a relative term, simply denoting the absence of complex architectural patterns as described earlier. Exaggerated apical cytoplasmic snouts and abundant flocculent intraluminal secretions are often present, and some of the cells comprising such lesions may have a hobnail appearance. These lesions frequently show intraluminal calcifications,

\section{Figure 1}
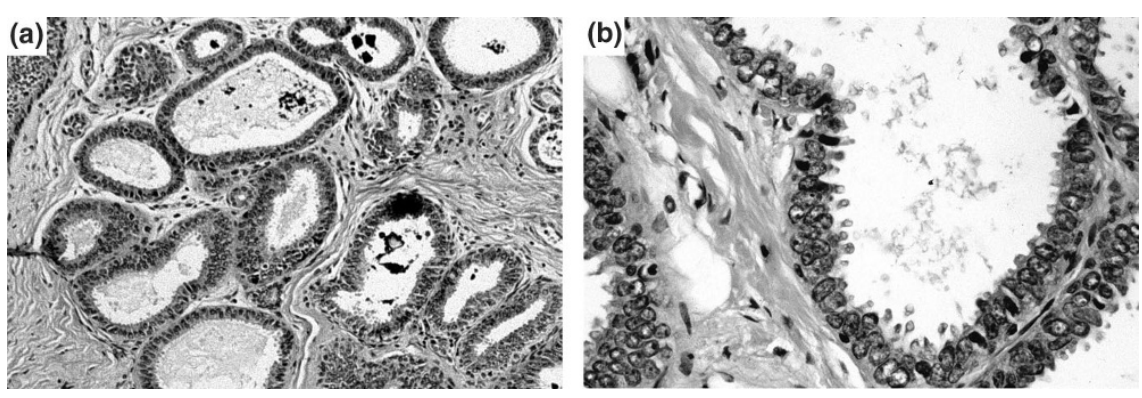

Flat epithelial atypia: columnar cell change with atypia. (a) This terminal duct lobular unit shows variably dilated acini, many of which contain intraluminal secretions and calcifications. Most acini are lined by only one or two layers of columnar epithelial cells, many of which show prominent apical cytoplasmic snouts (hematoxylin \& eosin, original magnification 10x). (b) The columnar epithelial cells lining the acini show cytologic atypia, characterized by enlarged, monotonous nuclei; the nuclear/cytoplasmic ratio is increased. Nucleoli are evident in some of the nuclei (hematoxylin \& eosin, original magnification $40 x$ ). 
Figure 2

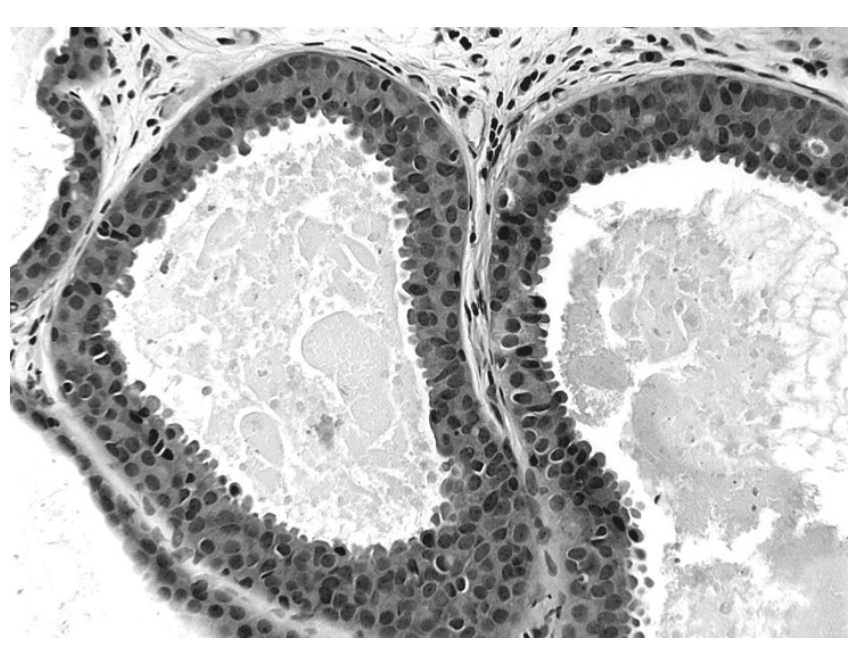

Flat epithelial atypia: columnar cell hyperplasia with atypia. The acini in this terminal duct lobular unit are lined by a few layers of columnar epithelial cells that show low-grade cytologic atypia, characterized by relatively round, monotonous nuclei (hematoxylin \& eosin, original magnification 20x).

which in some instances may have the configuration of psammoma bodies.

High-grade cytologic atypia with nuclear pleomorphism of the type seen in high-grade DCIS is not a feature of lesions we include in the categories of columnar cell change with atypia or of columnar cell hyperplasia with atypia [12]. The presence of such high-grade nuclear features merits the designation of high-grade DCIS, even if the cells comprise only a single cell layer [6]. However, such lesions are rarely seen in the absence of high-grade DCIS exhibiting other architectural patterns.

It is important to note that while the flat lesions we have designated columnar cell change with atypia and columnar hyperplasia with atypia may be observed in isolation, they often coexist with more complex proliferative lesions that are composed of cytologically similar cells and that fulfill the diagnostic criteria for ADH or DCIS. In fact, their presence should prompt a diligent search for such areas.

\section{Biological markers}

Studies of the expression of various biological markers in flat epithelial atypia are fairly limited. The available data have indicated that the majority of the cells comprising these lesions exhibit expression of cytokeratin 19 [13], and they consistently lack expression of high molecular weight cytokeratins as defined by antibody 34 beta E-12 and antibodies to cytokeratin 5 and cytokeratin 6 [14] (Carlo V, Fraser J, Pliss N, Connolly JL, Schnitt S, unpublished data, 2003). In addition, the cells typically show intense nuclear expression of the estrogen receptor
$[3,15,16]$ and the progesterone receptor [13] in the majority of the cells. These cells also show strong cytoplasmic expression of the bcl-2 protein [15] and variable expression of cyclin $D_{1}$ [13]. In most examples of flat epithelial atypia, very few of the cells show staining for the Ki-67 antigen, indicating that the cells comprising these lesions have a low proliferative rate [15].

\section{Clinical significance}

Assessment of the clinical significance of the flat epithelial atypias has been hampered by variations in the terminology used to describe these lesions and by the limited number of cases that have been studied in a systematic fashion. There have been two types of studies that have addressed the potential clinical importance of these lesions: observational studies and clinical outcome studies.

\section{Observational studies}

A number of authors have noted that flat epithelial atypias are often seen in association with DCIS and with some types of invasive breast carcinoma, particularly tubular carcinoma. Oyama and colleagues studied 21 cases of the flat lesion they termed "atypical cystic lobules" [3], and found these lesions to be more common in specimens containing DCIS than in specimens without DCIS $(36 \%$ versus 3\%). They also noted geographic proximity between the flat lesion and the DCIS. Weidner described a lesion composed of small ectatic ducts lined by one or two layers of columnar cells with apical snouts similar to those seen in tubular carcinoma [17]. He considered this flat lesion to represent an example of low-grade DCIS. In a more detailed analysis, Goldstein and O'Malley found an association between a similar lesion they called "small ectatic ducts lined by atypical cells with apocrine snouts" with both low-grade DCIS and tubular carcinoma [11]. Other authors have also noted an association between various flat atypical lesions and DCIS and/or invasive carcinoma $[4,9,10]$.

In addition, cytologic, immunophenotypic, and genetic similarities between flat epithelial atypias and DCIS and/or tubular carcinoma have been described by various authors.

Among 16 cases with both atypical cystic lobules and low-grade DCIS, Oyama and colleagues noted that the cells comprising the atypical cystic lobules were cytologically similar to the cells of the fully developed DCIS present in the same specimen [3]. Other investigators have also noted that the cells comprising some flat atypical lesions are cytologically similar to or identical to the cells comprising some forms of DCIS or to the cells comprising the glands of tubular carcinoma $[6,8,10,11,18]$. Oyama and colleagues further noted an immunophenotypic identity between atypical cystic lobules and coexistent low-grade DCIS (the cells of both lesions being 
Table 2

Outcome of patients with 'clinging carcinoma' (flat type, with low nuclear grade/monomorphic nuclei)

\begin{tabular}{|c|c|c|}
\hline & Eusebi and colleagues [6] & $\begin{array}{l}\text { European Organization for Research and } \\
\text { Treatment of Cancer Trial } 10853 \text { [19] }\end{array}$ \\
\hline Number of patients & 25 & 59 \\
\hline Type of study & $\begin{array}{l}\text { Retrospective review of breast biopsies } \\
\text { originally considered benign }\end{array}$ & Prospective, randomized clinical trial \\
\hline Treatment & Diagnostic biopsy; no attempt at excision & Excision alone or excision and radiation therapy \\
\hline Follow-up & 19.2 years (mean) & 5.4 years (median) \\
\hline Number (\%) with local recurrence & $1(4 \%)^{a}$ & 0 \\
\hline $\begin{array}{l}\text { Number with subsequent invasive } \\
\text { breast cancer }\end{array}$ & 0 & 0 \\
\hline
\end{tabular}

positive for the estrogen receptor, the progesterone receptor, keratin 19 and cyclin $D_{1}$ ) in their 16 cases [3]. In a recent genetic study of 13 cases of the flat atypical lesion designated 'DIN-flat monomorphic type', Moinfar and colleagues found that $70 \%$ showed loss of heterozygosity at one or more of the eight loci evaluated [8]. Furthermore, they found that the genetic alterations in these columnar cell lesions were the same as those in the associated DCIS or invasive cancer [8].

Based on the foregoing observations, it is reasonable to conclude that at least some flat epithelial atypias are probably neoplastic proliferations that may well represent either a precursor of or the earliest morphologic manifestation of low-grade DCIS, as well as being a precursor to invasive carcinoma, particularly tubular carcinoma. While this is of great interest from a biological point of view, the clinical implications of these observations can only be determined from follow-up studies. Unfortunately there is a paucity of data on this subject, and the few available studies are characterized by very small numbers of patients.

\section{Clinical outcome studies}

Only two follow-up studies have directly addressed the clinical significance of flat epithelial atypia to date. In a review of more than 9000 breast biopsies that were initially considered benign, Eusebi and colleagues retrospectively identified 25 patients with so-called "clinging carcinoma" of the flat, monomorphic (low nuclear grade) type [6]. Only one of these patients (4\%) is reported to have developed a 'local recurrence' after an average follow-up period of 19.2 years. However, the local recurrence in this patient consisted of a clinging carcinoma lesion histologically identical to the original lesion, and it is not possible to determine whether this simply reflected sion or whether this represented a true local recurrence. Of note, none of these 25 patients developed an invasive breast cancer within the follow-up period.

In another study, 59 patients with clinging carcinoma of the low nuclear grade type were identified among the patients entered into European Organization for Research and Treatment of Cancer trial 10853, a randomized clinical trial comparing excision and radiation therapy with excision alone for the treatment of women with DCIS [19]. None of these 59 patients developed a local recurrence or an invasive breast cancer with a median follow-up period of 5.4 years. The very limited available data thus suggest that, among patients with so-called clinging carcinoma of the low nuclear grade/monomorphic type (lesions that we would categorize as columnar cell change with atypia or columnar cell hyperplasia with atypia), the likelihood of local recurrence or progression to invasive breast cancer is exceedingly low, at least with the available follow-up (Table 2). Additional clinical follow-up studies are clearly needed, however, to better understand the relationship between these flat epithelial atypias and the risk of subsequent breast cancer.

\section{Practical considerations}

The appropriate pathology work-up and clinical management of patients whose biopsy specimens show flat epithelial atypias are evolving as information regarding these lesions begins to accumulate. The limited available data suggest that when flat epithelial atypia is encountered in a core needle biopsy specimen, subsequent excision shows a more advanced lesion in about one-third of cases. This is sufficiently frequent to recommend excision in such cases as a matter of routine $[20,21]$.

The presence of flat epithelial atypia in an excisional biopsy specimen should prompt a careful search for areas 
with diagnostic features of $\mathrm{ADH}$ or $\mathrm{DClS}$, by examining additional sections from the block or blocks containing the lesion and by the submission of the remainder of the tissue for histological examination. There are several other considerations regarding the identification of flat epithelial atypia in excisional biopsy specimens that merit discussion. When a proliferation that fulfills the diagnostic criteria for ADH or DCIS is found to arise in a background of flat epithelial atypia, it seems most prudent to manage the patient as one would manage ADH or DCIS in any other setting. However, there are two issues that remain to be resolved when the flat atypia is found to coexist with diagnostic areas of DCIS, particularly in cases in which the cytologic features of the cells comprising the flat atypia are similar to those of the cells comprising the diagnostic areas of DCIS. The first issue is whether the flat atypia should be taken into consideration in determining the size or the extent of the DCIS lesion. The second is whether the presence of flat atypia at the excision margins is sufficient to render the margins 'positive', requiring further surgical resection. As noted earlier, the limited available clinical data suggest that flat atypias are associated with a very low risk of recurrence or progression to invasive carcinoma. Therefore, we believe that these lesions should not be taken into consideration when determining the size of a coexistent DCIS lesion or in the evaluation of the status of the margins of excision, even when they are composed of cells that are cytologically similar to those in the diagnostic areas of DCIS [12].

Another problem is the management of patients whose breast biopsies, after thorough examination, show flat epithelial atypia (columnar cell change with atypia and/or columnar cell hyperplasia with atypia) without diagnostic areas of ADH or DCIS. Again, pathologists that consider these flat lesions to represent 'cancerization of lobules' by the cells of low-grade DCIS or 'clinging carcinoma' would argue that they should be considered variants of DCIS, and managed as such [11]. Others would argue that, despite the fact that these flat lesions may well be neoplastic and may even be composed of cells that are identical to those seen in some forms of DCIS or even tubular carcinoma, the few available clinical follow-up studies suggest that they are associated with a risk of subsequent breast cancer that is considerably lower than that seen with fully developed forms of low-grade DCIS. The concern, therefore, is that managing patients with such lesions as if they had DCIS would result in overtreatment of many patients.

\section{Conclusions}

Lesions included within the category of flat epithelial atypia (i.e. lesions that we currently designate columnar cell change with atypia and columnar cell hyperplasia with atypia) are being encountered with increasing frequency due to the widespread use of screening mammography.
This article is the fourth in a review series on The diagnosis and management of pre-invasive breast disease - current challenges, future hopes, edited by Sunil R Lakhani.

Other articles in the series can be found at http://breast-cancer-research.com/articles/reviewseries.asp?series=bcr_Thediagnosis

Recent studies have begun to provide insights into the biological and clinical significance of these lesions. However, additional morphologic, immunophenotypic and genetic studies are needed to better define the relationships between these flat atypical lesions to DCIS and invasive breast cancer. Emerging data suggest that these flat epithelial atypias may be neoplastic lesions that represent the earliest form of low-grade DCIS. Despite this, the limited available clinical follow-up data suggest that the risk of local recurrence or progression of these lesions to invasive cancer is quite low, supporting the notion that categorizing such lesions as 'clinging carcinoma' and managing them as if they were fully developed DCIS will result in overtreatment of many patients. Thus, at this time, the appropriate management of patients whose breast biopsies show flat epithelial atypia in the absence of diagnostic areas of $\mathrm{ADH}$ or DCIS is unknown and requires evaluation in further clinical outcome studies.

\section{Competing interests}

None declared.

\section{Acknowledgement}

The author thanks Dr Anne Vincent-Salomon, Institut Curie, Paris, France for many helpful discussions concerning this manuscript.

\section{References}

1. Tavassoli FA, Hoefler H, Rosai J, Holland R, Ellis I, Schnitt S, Lakhani, SR, Boecker W, Heywang-Kobrunner SH, Moinfar F, Peterse J: Intraductal proliferative lesions. In: Pathology and Genetics of Tumours of the Breast and Female Genital Organs. Edited by Tavassoli FA, Devilee P. Lyon: IARC Press; in press.

2. Tsuchiya S: Atypical ductal hyperplasia, atypical lobular hyperplasia, and interpretation of a new borderline lesion. Jpn J Cancer Clin 1998, 44:548-555.

3. Oyama T, lijima K, Takei H, Horiguchi J, lino Y, Nakajima T, Koerner F: Atypical cystic lobule of the breast: an early stage of low-grade ductal carcinoma in-situ. Breast Cancer 2000, 7: 326-331.

4. Wellings SR, Jensen HM, Marcum RG: An atlas of subgross pathology of the human breast with special reference to possible precancerous lesions. J Natl Cancer Inst 1975, 55:231-273.

5. Azzopardi JG: Problems in Breast Pathology. Philadelphia, PA: WB Saunders; 1979.

6. Eusebi V, Feudale E, Foschini MP, Micheli A, Conti A, Riva C, DiPalma S, Rilke F: Long-term follow-up of in situ carcinoma of the breast. Semin Diagn Pathol 1994, 11:223-235.

7. Fraser JL, Raza S, Chorny K, Connolly JL, Schnitt SJ: Columnar alteration with prominent apical snouts and secretions: a spectrum of changes frequently present in breast biopsies performed for microcalcifications. Am J Surg Pathol 1998, 22: 1521-1527.

8. Moinfar F, Man YG, Bratthauer GL, Ratschek M, Tavassoli FA: Genetic abnormalities in mammary ductal intraepithelial neo- 
plasia-flat type ('clinging ductal carcinoma in situ'): a simulator of normal mammary epithelium. Cancer 2000; 88:20722081.

9. Page DL, Kasami M, Jensen RA: Hypersecretory hyperplasia with atypia in breast biopsies. What is the proper level of clinical concern? Pathol Case Rev 1996, 1:36-40.

10. Rosen PP: Columnar cell hyperplasia is associated with lobular carcinoma in situ and tubular carcinoma [letter]. $\mathrm{Am} J$ Surg Pathol 1999, 23:1561.

11. Goldstein NS, O'Malley BA: Cancerization of small ectatic ducts of the breast by ductal carcinoma in situ cells with apocrine snouts: a lesion associated with tubular carcinoma. Am J Clin Pathol 1997, 107:561-566.

12. Schnitt SJ, Vincent-Salomon A: Columnar cell lesions of the breast. Adv Anat Pathol 2003, 10:113-124.

13. Oyama $T$, Maluf $H$, Koerner $F$ : Atypical cystic lobules: an early stage in the formation of low-grade ductal carcinoma in situ. Virchows Arch 1999, 435:413-421.

14. Otterbach F, Bankfalvi A, Bergner S, Decker T, Krech R, Boecker W: Cytokeratin 5/6 immunohistochemistry assists the differential diagnosis of atypical proliferations of the breast. Histopathology 2000, 37:232-240.

15. Fraser JL, Raza S, Chorny K, Connolly JL, Schnitt SJ: Immunophenotype of columnar alteration with prominent apical snouts and secretions (CAPSS) [abstract]. Lab Invest 2000, 80:21A.

16. Allred DC, Mohsin SK, Fuqua SA: Histological and biological evolution of human premalignant breast disease. Endocr Relat Cancer 2001, 8:47-61.

17. Weidner $\mathrm{N}$ : Malignant breast lesions that may mimic benign tumors. Semin Diagn Pathol 1995, 12:2-13.

18. Rosen PP: Rosen's Breast Pathology. Philadelphia, PA: Lippincott-Raven; 2001.

19. Bijker N, Peterse JL, Duchateau L, Julien JP, Fentiman IS, Duval C, DiPalma S, Simony-Lafontaine J, de Mascarel I, van de Vijver M: Risk factors for recurrence and metastasis after breast-conserving therapy for ductal carcinoma in-situ: analysis of European Organization for Research and Treatment of Cancer Trial 10853. J Clin Oncol 2001, 19:2263-2271.

20. Brogi E, Tan LK: Findings at excisional biopsy (EBX) performed after identification of columnar cell change (CCC) of ductal epithelium in breast core biopsy (CBX) [abstract]. Mod Pathol 2002, 15:29A-30A.

21. Harigopal M, Yao DX, Hoda SA, DeLellis RA, Vazquez MF: Columnar cell alteration diagnosed on mammotome core biopsy for indeterminate microcalcifications: results of subsequent mammograms and surgical excision [abstract]. Mod Pathol 2002, 15:36A.

\section{Correspondence}

Stuart J Schnitt, MD, Department of Pathology, Beth Israel Deaconess Medical Center, 330 Brookline Avenue, Boston, MA 02215, USA. Tel: +1 617667 4344; fax: +1 $617 \quad 975$ 5620; e-mail: sschnitt@bidmc.harvard.edu 